\title{
Un adénovirus tumoricide spécifique
}

L'adénovirus a besoin, pour se répliquer dans les cellules, de stimuler la machinerie de synthèse d'ADN. Il le fait principalement grâce à l'oncogène $\mathrm{E} 1 \mathrm{~A}$ dont le mode d'action est multiple et complexe. E1A agit notamment en se fixant à la protéine $\mathrm{Rb}$, qui libère alors l'activateur transcriptionnel E2F. De plus, E1A interagit avec les co-activateurs p300 et CBP $\left(m / s n^{\circ} 10\right.$, vol. 12, p. 1113). Cependant, la cellule possède plusieurs systèmes de sécurité permettant d'éviter une activation indue de la machinerie réplicative : l'inhibition de la synthèse de l'ADN et l'apoptose. La protéine p53 bloque la synthèse d'ADN et le cycle cellulaire en phase G1; elle induit également l'apoptose. Indépendamment de p53, d'autres systèmes peuvent également aboutir à cette mort cellulaire programmée. La région adénovirale E1B contrecarre le blocage de la réplication et l'apoptose par l'intermédiaire de deux protéines pour lesquelles elle code. La protéine $55 \mathrm{~K}$ se fixe à p53 et l'inactive. La protéine $19 \mathrm{~K}$, un équivalent fonctionnel de Bcl2, inhibe l'apoptose. C'est fort de ces notions que Franck Mc Cormick et ses collègues, de la firme ONYX (Richmond, CA, USA) ont testé le pouvoir anticancéreux d'un mutant adénoviral ayant à la fois une délétion de 827 paires de bases dans la région $\mathrm{E} 1 \mathrm{~B}$ de liaison à $\mathrm{p} 53$ et une mutation stop empêchant la synthèse d'une protéine tronquée. Ce mutant ne synthétise pas E1B $55 \mathrm{~K}$, sans modification de E1B 19K. De ce fait, ce virus, incapable d'inactiver p53, ne peut se répliquer dans des cellules normales vis-à-vis desquelles il est donc inoffensif. En revanche, la réplication est pratiquement normale dans des cellules déficientes en p53 qui sont ainsi lysées par effet cytopa- également au niveau de cellules de cancer cervical humain greffées chez la souris immunodéprimée nude [1]. Forte de ces résultats, e t des contrôles démontrant l'apparente innocuité de ce virus, la FDA (food and drug administration) a autorisé des essais cliniques de phase I chez une douzaine de malades. Les premiers résultats indiquent que le traitement est bien toléré, même au plus fort titre de virus, mais il est naturellement bien trop tôt pour savoir si la stratégie est efficace dans des cancers humains. Les limitations que l'on peut envisager à cette stratégie sont en effet multiples. Tout d'abord, le traitement nécessite une infection directe de la tumeur, par injection locale, ce qui n'est possible qu'au niveau de cancers facilement accessibles. D'ailleurs les essais cliniques concernent des cancers de la tête et du cou. Ensuite, les malades ne sont pas immunodéprimés, comme le sont les souris nude et ils possèdent presque tous des anticorps anti-adénovirus qui pourraient rendre inefficace la propagation de l'infection adénovirale aux cellules cancéreuses. Enfin, la spécificité de ce virus pourrait être inférieure chez l'homme à ce qu'elle semble être chez la souris.

En effet, les rongeurs ne sont pas permissifs pour la réplication d'adénovirus qui ne peut donc se faire que dans les cellules tumorales humaines greffées, déficientes en p53. En revanche, certains tissus humains pourraient être sensibles à l'adénovirus malgré l'absence de la séquence codant pour E1B 55K. C'est le cas de certaines cellules embryonnaires et pourrait l'être également, à un certain niveau, d'autres cellules en division rapide. En effet, la division cellulaire active nécessite peu ou prou une inactivation fonctionnelle de p53. Il n'empêche que cette stratégie est extrêmement élégante et qu'elle pourrait ne constituer que la première étape d'une recherche tendant à préparer des virus inoffensifs doués de vraies actions antitumorales. Un tel outil correspondrait, naturellement, au rêve de tous les cancérologues: un moyen thérapeutique inoffensif vis-à-vis des cellules normales et actif sur une grande diversité de tissus cancéreux !

A.K.

1. Bischoff JR, Kirn DH, Williams A, Heise C, Horn S, et al. An adenovirus mutant that replicates selectively in p53-deficient human tumor cells. Science 1996; 274 : 373-6.

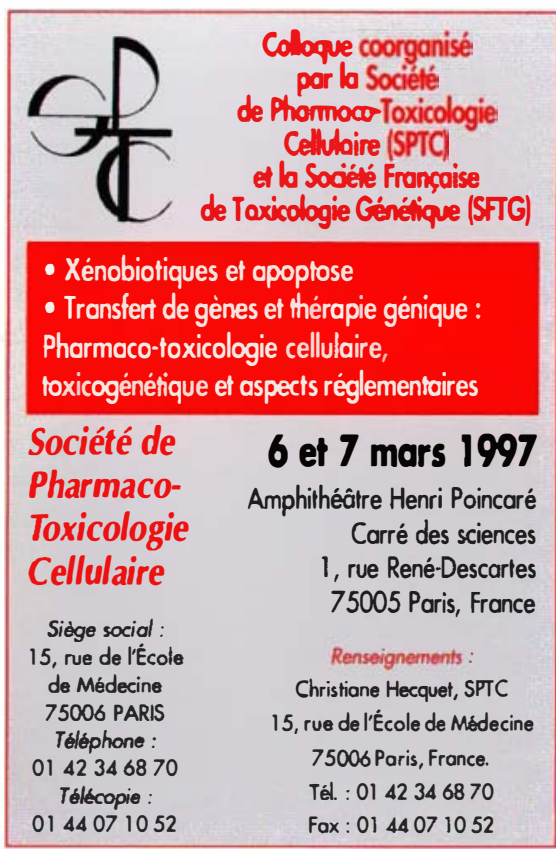

$m / s n^{\circ} 1$, vol. 13, janvier 97 


\section{La résistance au paludisme. Interaction de facteurs multiples}

Si la notion d'une action à la fois sélective et protectrice exercée par le paludisme sur les hémoglobinopathies repose sur un ensemble incontestable de données épidémiologiques, le mécanisme en cause est encore très mal connu malgré le grand nombre d'études focalisées sur le sujet. Un travail récent, impliquant plusieurs équipes, mais coordonné par celle de John Clegg (Oxford, GB) apporte à cette compréhension une contribution importante [1]. La même équipe, étudiant l'épidémiologie du paludisme à Plasmodium falciparum en Mélanésie, avait pu établir une corrélation très étroite, dans laquelle interviennent latitude et altitude, entre endémie palustre et $\alpha$-thalassémie, rendant plus que vraisemblable l'hypothèse d'une sélection par le parasite [2]. Le présent travail, appuyé sur des données de biologie moléculaire, a été mené dans une région du Vanuatu où la fréquence génique de l' $\alpha$-thalassémie délétionnelle est 0,26 , et le paludisme hyperendémique, dû tant au $P$. falciparum, qu'au $P$. vivax. Chez plus de 900 enfants de moins de 10 ans, le génotype a été déterminé et la fréquence d'épisodes cliniques fébriles de paludisme relevée. A leur surprise, les auteurs ont constaté une fréquence nettement accrue $(\mathrm{p}<0,005)$ chez les enfants homozygotes pour la délétion $(\alpha-/ \alpha-)$. Cette prédisposition n'était observée qu'avant 5 ans, elle existait vis-à-vis des deux parasites et s'accompagnait d'une splénomégalie supérieure à celle qu'induit l' $\alpha$-thalassémie seule. Chez les hétérozygotes $(\alpha \alpha / \alpha-)$ la fréquence des épisodes cliniques n'était pas augmentée, mais seule l'était celle d'une splénomégalie. La question se pose donc de $m / s n^{\circ} 1$, vol. 13 , janvier 97 réconcilier cette incidence accrue d'un paludisme à traduction clinique avec la preuve d'une protection contre les formes graves qu'ont apportée les données épidémiologiques. Les auteurs donnent à cette contradiction apparente une base immunologique. Comme dans tous les syndromes thalassémiques, il y a au cours des $\alpha$-thalassémies un cèrtain degré d'érythropoïèse inefficace, et donc une augmentation des cellules jeunes dans la circulation qui, métaboliquement plus actives, sont préférentiellement envahies par le $P$. falciparum; quant au $P$. vivax, il n'envahit que les réticulocytes. L'observation, par ailleurs, que la présentation de l'antigène parasitaire au système immunitaire, à la surface des érythrocytes, est double dans le globule rouge $\alpha$-thalassémique infesté de ce qu'elle est dans la cellule normale n'est pas sans intérêt de ce point de vue [3]. Il est loisible de penser que, pendant la petite enfance, l'enfant étant protégé par l'immunité maternelle et par un taux élevé d'hémoglobine fœtale, des infections répétées provoquent une immunité, qui protégera ultérieurement contre les formes graves. L'hypothèse d'une immunité croisée par le $P$. vivax est évoquée, liée à une infestation de l'enfant $\alpha$ thalassémique par ce parasite plus précoce dans l'existence, et apportant une protection limitée mais réelle contre les formes graves ultérieures dues à $P$. falciparum [4]. Cependant, cette hypothèse semble en contradiction avec le fait que le $P$. vivax, qui a eu des usages thérapeutiques, ne s'est pas toujours avéré protecteur contre le P. falciparum.

Les phénomènes de résistance au paludisme, et la sélection qui en est la conséquence, sont d'importance majeure. L'équipe d'Oxford avait montré le rôle de certains antigènes HLA $\left(\mathrm{m} / \mathrm{s} n^{\circ} 9\right.$, vol 7, p. 981) et ultérieurement identifié à la phase hépatique du parasite un nonapeptide reconnu spécifiquement par les lymphocytes $T$ cytotoxiques spécifiques de HLA-B53 et pouvant servir de base à une vaccination [5]. Le rôle régulateur de cytokines a aussi pu être montré: une mutation du promoteur du TNF- $\alpha$ est lié en Gambie à une susceptibilité au paludisme cérébral [6]. Par conséquent, les mécanismes de la sensibilité ou de la résistance au paludisme sont, à l'évidence, plurigéniques, nécessitant des recherches pluridisciplinaires et complexes, amplement justifiées par la gravité de cette maladie et les perspectives attendues des progrès accomplis.

D.L.

1. Williams TN, Maitland K, Bennett S, Ganczakowski M, Peto TEA, et al. High incidence of malaria in $\alpha$-thalassemic children. Nature 1996; 383: $522-5$.

2. Flint J, Hill AV, Bowden DK, Oppenheimer SJ, Sill PR, et al. High frequencies of alpha-thalassaemia are the result of natural selection by malaria. Nature 1986; 321 : 744-50.

3. Luzzi GA, Merry AH, Newbold CI, Marsh K, Pasvol G, Weatherall DJ, et al. Surface antigen expression on Plasmodium falciparum-infected erythrocytes is modified in alpha- and beta-thalassemia. J Exp Med 1991 ; 173 : 785-91.

4. Miller LH. Protective selective pressure. Nature $1996 ; 383: 480-1$.

5. Hill AVS, Elvin J, Willis AC, Aidoo M, Allsopp $\mathrm{CE}$, et al. Molecular analysis of the association of HLA-B53 and resistance to severe malaria. Nature 1992 ; 360 : 434-9.

6. McGuire W, Hill AVS, Allsopp CE, Greenwood $\mathrm{BM}$, Kwiatkowski D. Variation in the TNF- $\alpha$ promoter region associated with susceptibility to cerebral malaria. Nature 1994 ; 371 : 508-11. 